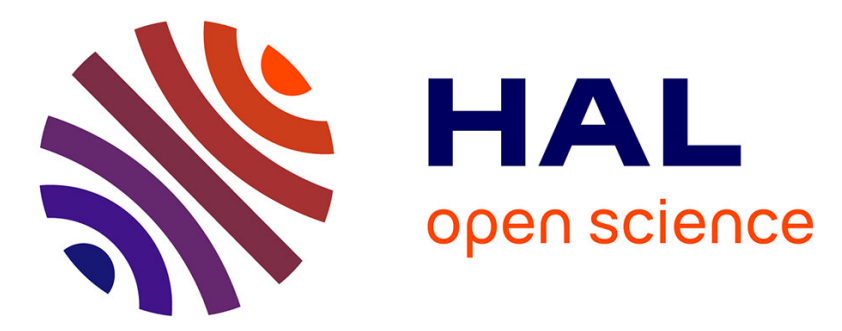

\title{
Approximate Sum-Capacity of Full- and Half-Duplex Asymmetric Interference Channels with Unilateral Source Cooperation
}

\author{
Martina Cardone, Daniela Tuninetti, Raymond Knopp, Umer Salim
}

\section{To cite this version:}

Martina Cardone, Daniela Tuninetti, Raymond Knopp, Umer Salim. Approximate Sum-Capacity of Full- and Half-Duplex Asymmetric Interference Channels with Unilateral Source Cooperation. Information Theory and Applications Workshop, Feb 2013, United States. pp.1-9. hal-00875049

\author{
HAL Id: hal-00875049 \\ https://hal.science/hal-00875049
}

Submitted on 21 Oct 2013

HAL is a multi-disciplinary open access archive for the deposit and dissemination of scientific research documents, whether they are published or not. The documents may come from teaching and research institutions in France or abroad, or from public or private research centers.
L'archive ouverte pluridisciplinaire HAL, est destinée au dépôt et à la diffusion de documents scientifiques de niveau recherche, publiés ou non, émanant des établissements d'enseignement et de recherche français ou étrangers, des laboratoires publics ou privés. 


\title{
Approximate Sum-Capacity of Full- and Half-Duplex Asymmetric Interference Channels with Unilateral Source Cooperation
}

\author{
Martina Cardone ${ }^{\dagger}$, Daniela Tuninetti* ${ }^{*}$ Raymond Knopp ${ }^{\dagger}$, Umer Salim $^{\ddagger}$ \\ $\dagger$ Eurecom, Sophia Antipolis, 06560, France, Email: \{cardone, knopp\}@eurecom.fr \\ * University of Illinois at Chicago, Chicago, IL 60607, USA, Email: danielat@uic.edu \\ $\ddagger$ Intel Mobile Communications, Sophia Antipolis, 06560, France, Email: umer.salim@intel.com
}

\begin{abstract}
This paper focuses on the causal cognitive radio channel. This model consists of a two-user Gaussian interference channel where a cooperative secondary/cognitive source can causally learn the message of a primary source through a noisy link. The generalized Degrees-of-Freedom (gDoF) and the sumcapacity to within a constant gap are characterized for channels where one destination does not experience interference, referred to as interference-asymmetric channels. Both cases where the cognitive source operates in full- and half-duplex mode are considered. The different models are compared among themselves and with the case of interference-symmetric channels with either bilateral or unilateral source cooperation. In particular, the parameter regimes where causal cognition, or unilateral cooperation, attains the same $\mathrm{gDoF}$ of classical channel models, such as the noncooperative interference channel and the noncausal cognitive channel, are identified in order to determine when causal cognitive radio offers benefits in practical systems.
\end{abstract}

\section{INTRODUCTION}

In this work we consider the Gaussian Interference Channel (IC) with unilateral source cooperation, which consists of two source-destination pairs that communicate over a shared wireless channel. In this model, each pair aims to reliably communicate at a certain rate and the two transmissions interfere with each other. Moreover, a primary user/user 1 is aided by a secondary user/user 2 to convey data to its intended destination. As opposed to the classical cognitive channel [1], where the cognitive source is assumed to have full non-causal a priori knowledge of the primary's message, here for practical reasons we impose that the secondary user learns the primary's message causally over a noisy link. This channel model is a special case of the IC with generalized feedback [2], [3], [4], or source cooperation, where only one source engages in cooperation. Understanding the fundamental performance limits of this channel is critical to the deployment of future wireless cognitive networks as it indicates whether substantial gains are possible over the noncooperative case and whether the performance of the idealistic non-causal cognitive channel can indeed be attained.

The interference-symmetric IC with source cooperation, wherein both destinations experience interference, has been widely studied in the literature [2], [3], [4], [5], [6]. In this work we study the interference-asymmetric IC, where either the link from source 1 to destination 2 is nonexistent (Zchannel) or the link from source 2 to destination 1 is nonexistent (S-channel). Due to the asymmetry in the cooperation, the Z-channel and the S-channel have different capacities and we analyze them separately. In practice, interference-asymmetric models capture scenarios where one destination is in the coverage area of only one of the sources, see Fig. 1.

For the noncooperative Z-channel much more is known compared to the general interference-symmetric IC. For example, the sum-capacity of the Z-channel is known for all channel parameters [7], while for the general interference-symmetric IC the sum-capacity is still an open problem in the weak interference regime [8]. Moreover, the sum-capacity achieving scheme for the Z-channel does not require rate splitting, as opposed to the general interference-symmetric IC. Finally, the sum-capacity of the Z-channel is larger than that of the general interference-symmetric IC because of the reduced interference at one destination. In this work we consider unilateral source cooperation and we aim to understand whether the absence of interference at one destination improves performance or simplifies the achievable schemes compared to the interferencesymmetric IC. Our result sheds light into the ultimate limits of practical cognitive radio channels as a function of the network topology and might guide the node placement in future systems.

\section{A. Past Work}

The presence of communication links between the sources enables cooperation. An IC with unilateral cooperation, or causal cognitive radio, is a special case of the general IC with bilateral source cooperation, or generalized feedback, where one cooperation link is nonexistent. The capacity of the IC with unilateral cooperation is lower bounded by that of the noncooperative IC [8] and upper bounded by that of the noncausal cognitive IC [9], both known to within 1 bit.

Two modes of operation for the cooperative cognitive source are considered: Full-Duplex (FD) and Half-Duplex (HD). A node operates in FD mode if it can receive and transmit over the same time-frequency resource, and in HD mode otherwise.

The Full-Duplex Interference-Symmetric Channel. HostMadsen first studied outer and inner bounds for the Gaussian 

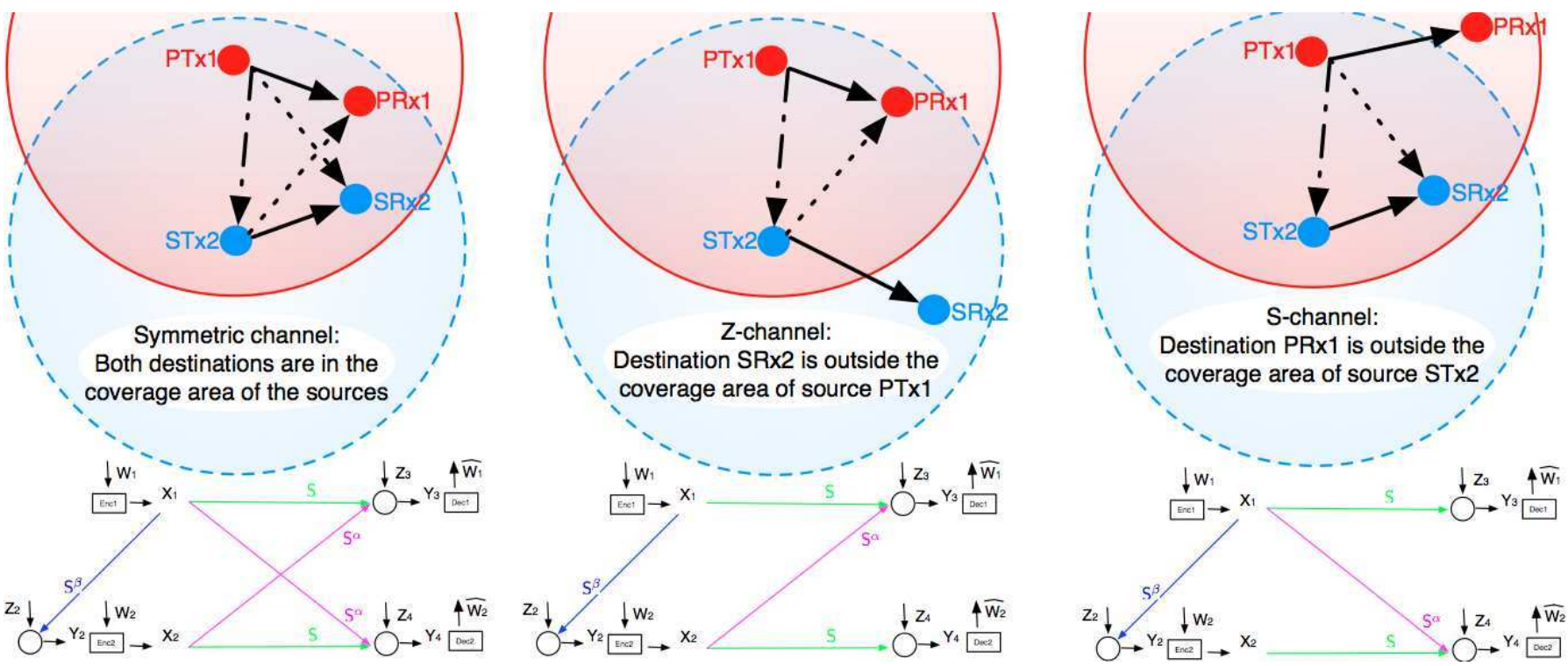

Fig. 1: The Gaussian Interference Channel with Unilateral Source Cooperation. Left: symmetric channel. Center: Z-channel. Right: S-channel.

IC with either source or destination cooperation [2]. A novel sum-rate outer bound for the "semi-deterministic injective" IC with source cooperation was developed in [4, eq.(7)] as an extension of [10, eq.(3)]. A novel sum-rate outer bound for the general memoryless IC with source cooperation appeared in [11, eqs.(4d)-(4e)] as an extension of [12, eq.(19)]. The largest known achievable region for the general IC with source cooperation, to the best of our knowledge, is [3]. With these bounds, the sum-capacity of the FD Gaussian IC with bilateral symmetric cooperation (i.e., by channel reciprocity the cooperation links between the sources are assumed to have the same strength) but with otherwise generic direct and cross/interference links, was characterized to within 20 bits in [4]. The gap was reduced to 4 bits in [13] for the case of symmetric cooperation and symmetric interference links.

Since causal cognition/unilateral FD cooperation assumes that one cooperation link is absent, capacity results to within a constant gap for unilateral FD cooperation are not implied by the gap result in [4]. However, bounds valid for a general memoryless IC with bilateral FD source cooperation can be straightforwardly adapted to the case of unilateral FD cooperation. We followed this approach in [6] and characterized the sum-capacity to within 7.3 bits for the interference-symmetric case (model depicted on the left in Fig. 1).

Cognitive channels were also studied in [14]; here the authors analyzed the causal cognitive IC with delay. The zerodelay case coincides with the causal cognitive model studied in this work; the main contribution is a capacity characterization for the degraded semi-deterministic channel in the strong interference regime.

The Half-Duplex Interference-Symmetric Channel. The case where nodes operate in HD mode can be analyzed within the framework of 'general memoryless IC with bilateral FD source cooperation' by introducing a state random variable that indicates whether a HD node is in receive- or in transmit- mode [15]. In [15] it was pointed out that higher rates can be achieved by considering random switch at the HD nodes compared to the case of deterministic switch. Here, deterministic switch refers to the case where the time and duration of the receive and transmit phases of each HD node is fixed a priori and known to all other nodes; when this is not the case, we say that a HD node uses a random switch to convey information to a destination (through the random times and durations of the receive and transmit phases). To the best of our knowledge, past work on the IC with HD cooperation has only considered the case of deterministic switch. A contribution of this work is to consider cooperative ICs with HD nodes that use random switch and quantify how much information can be carried by a random switch.

The case of HD source cooperation has received less attention compared to the FD case. In [5], the authors studied the IC with HD bilateral and unilateral source cooperation and with deterministic switch. For HD bilateral cooperation, the sum-capacity of the Gaussian noise channel with symmetric cooperation, direct and cross links was characterized to within $17+3=20$ bits [5, Thm.3.1]. For HD unilateral cooperation, the "cognitive capacity" (which is a sum-capacity achieving point that does not necessarily correspond to a corner point of the capacity region) with generic direct and cross links was characterized to within $23+2 \times 7=37$ bits [5, Thm.3.2]. In both cases, the faction of time the cooperative source listens to the channel and the gDoF were not given in closed form but as a solution of a linear program. In this paper we will extend the work of [5] by considering random switch and by analytically finding the gDoF in closed form.

Unilateral source cooperation was also considered in [16], [17] where the authors studied FD and/or HD cooperation with deterministic switch by developing several achievable schemes and evaluating them in Gaussian noise. However, capacity guarantees in terms of constant gap results were not given. 
The Full-Duplex Interference-Asymmetric Channel. In this work, we refer to an IC in which one destination does not experience interference as an interference-asymmetric channel. In the literature, this channel model is usually known as the Z-channel. For the Gaussian IC with symmetric bilateral FD cooperation (Fig. 1, left), the sum-capacity to within 20 bits follows from [4], which considered symmetric cooperation links but generic direct and cross links. For unilateral cooperation, two interference-asymmetric scenarios are possible, as shown in Fig. 1. To distinguish among them, we refer to them as the Z- (Fig. 1, middle) and the S-channel (Fig. 1, left).

To the best of our knowledge the Gaussian FD Z-channel has not been considered in the literature. The Gaussian FD S-channel was studied in [18] where a capacity corner point was determined for the case of weak cooperation and weak interference, but no 'capacity approximations' were given in other regimes. In this work we characterize the $\mathrm{gDoF}$ and the sum-capacity to within a constant gap for both the Z- and S-channel with unilateral FD cooperation.

The Half-Duplex Interference-Asymmetric Channel. For the Gaussian IC with unilateral HD cooperation with deterministic switch, the sum-capacity to within 20 bits follows from [5, Thm.3.2] for both the Z- and the S-channel, since [5, Thm.3.2] considered generic direct and cross links. However, [5, Thm.3.2] did not give closed-form expression for the gDoF and only considered deterministic switch. In this work we characterize the gDoF and the sum-capacity to within a constant gap for both the Z- and S-channel with unilateral HD cooperation and random switch by giving a closed-form expression for the gDoF and by substantially reducing the gap to no more than 5 bits.

\section{B. Contributions and Paper Outline}

In Section II the two-user Gaussian IC (G-IC) with source cooperation, where the cooperative source can operate either in FD or in HD mode, is introduced. The HD constraint is incorporated into the framework of memoryless FD framework by following [15]. In Section III we summarize known results for the interference-symmetric G-IC with source cooperation. In particular, in Section III-B we extend [5, Thm.3.1] to the case of random switch and provide a closed form expression for the gDoF. In Section IV we derive in closed-form the gDoF and characterize the sum-capacity to within a constant gap for the interference-asymmetric G-IC with unilateral source cooperation. Both the Z- and the S-channel are considered, as well as, FD and HD operation modes for the cognitive source. For HD cooperation, we extend the work of [5, Thm.3.2] to random switch and we reduce the gap. We also highlight when the ultimate performance of the considered models in terms of $\mathrm{gDoF}$ is the same as that of the noncooperative IC, or the Relay Channel, or the non-causal cognitive IC, and systematically discuss the impact of the network topology (i.e., differences between the interference-symmetric and the interference-asymmetric scenarios) on the $\mathrm{gDoF}$.

\section{SYSTEM MODEL}

A. The general memoryless interference channel with bilateral full-duplex source cooperation

An IC with source cooperation consists of two input alphabets $\left(\mathcal{X}_{1}, \mathcal{X}_{2}\right)$, four output alphabets $\left(\mathcal{Y}_{1}, \mathcal{Y}_{2}, \mathcal{Y}_{3}, \mathcal{Y}_{4}\right)$ and a memoryless transition probability $\mathbb{P}_{Y_{1}, Y_{2}, Y_{3}, Y_{4} \mid X_{1}, X_{2}}$. Source $j$ node $j, j \in\{1,2\}$, has a message $W_{j} \in\left[1: 2^{N R_{j}}\right]$ for destination $j$ /node $(j+2)$, where $N$ denotes the codeword length and $R_{j} \geq 0$ the transmission rate measured in bits per channel use. The messages $W_{1}$ and $W_{2}$ are independent and uniformly distributed on their respective domains. At time $t \in[1: N]$, source $j, j \in\{1,2\}$, sends $X_{j, t}\left(W_{j}, Y_{j}^{t-1}\right)$. At time $N$, destination $j, j \in\{1,2\}$, makes an estimate of its intended message as $\widehat{W}_{j}\left(Y_{j+2}^{N}\right)$. The capacity region is the convex closure of all non-negative rate pairs $\left(R_{1}, R_{2}\right)$ such that $\max _{j \in\{1,2\}} \mathbb{P}\left[\widehat{W}_{j} \neq W_{j}\right] \rightarrow 0$ as $N \rightarrow+\infty$.

\section{B. The Gaussian IC with source cooperation}

A single-antenna IC with bilateral source cooperation has input/output relationship

$$
\left[\begin{array}{l}
Y_{1} \\
Y_{2} \\
Y_{3} \\
Y_{4}
\end{array}\right]=\mathbf{H}\left[\begin{array}{l}
X_{1} \\
X_{2}
\end{array}\right]+\left[\begin{array}{l}
Z_{1} \\
Z_{2} \\
Z_{3} \\
Z_{4}
\end{array}\right]
$$

where the inputs are subject to a unitary average power constraint, the noises are independent proper-complex Gaussian random variables with zero mean and unit variance, and $\mathbf{H}$ is the constant complex-valued channel matrix that defines the connectivity of the network.

For FD source cooperation the channel matrix $\mathbf{H}$ is

$$
\mathbf{H}^{(\mathrm{FD})}:=\left[\begin{array}{cc}
\star & \sqrt{C_{1}} \\
\sqrt{C_{2}} & \star \\
\sqrt{S_{3}} & \sqrt{I_{3}} \mathrm{e}^{\mathrm{j} \theta_{3}} \\
\sqrt{I_{4}} \mathrm{e}^{\mathrm{j} \theta_{4}} & \sqrt{S_{4}}
\end{array}\right],
$$

for non-negative $\left(C_{1}, C_{2}, S_{3}, I_{3}, \theta_{3}, S_{4}, I_{4}, \theta_{4}\right)$ and where $\star$ indicates the channel gains that do not affect the capacity region (since a source can remove its transmit signal from its received signal). At each node, a channel gain can be taken to be real-valued and non-negative because a node can compensate for the phase of one of its channel gains.

For HD source cooperation the channel matrix $\mathbf{H}$ is

$$
\mathbf{H}^{(\mathrm{HD})}:=\left[\begin{array}{cccc}
1-S_{1} & 0 & 0 & 0 \\
0 & 1-S_{2} & 0 & 0 \\
0 & 0 & 1 & 0 \\
0 & 0 & 0 & 1
\end{array}\right] \mathbf{H}^{(\mathrm{FD})}\left[\begin{array}{cc}
S_{1} & 0 \\
0 & S_{2}
\end{array}\right]
$$

for the same $\mathbf{H}^{(\mathrm{FD})}$ in (2) since a HD channel is a special case of the memoryless FD framework [15] where the channel input at source $j, j \in\{1,2\}$, is now the pair $\left(X_{j}, S_{j}\right)$, where $X_{j} \in \mathcal{X}_{j}$ as before and where $S_{j} \in\{0,1\}$ is the state random variable that indicates whether the source is receiving $\left(S_{j}=0\right)$ or transmitting $\left(S_{j}=1\right)$.

An IC with unilateral cooperation is obtained with either $C_{1}=0$ or $C_{2}=0$, a Z-channel with $I_{4}=0$ and an S-channel with $I_{3}=0$. 
C. Performance Metrics: Generalized degrees-of-freedom and Sum-Capacity to within a Constant Gap

Let SNR $>0$ and consider the parameterization

$$
\begin{aligned}
S_{3} & =S_{4}:=\mathrm{SNR}, \\
I_{4} & :=\mathrm{SNR}^{\beta_{4}}, \beta_{4} \geq 0, \\
I_{3} & :=\mathrm{SNR}^{\beta_{3}}, \beta_{3} \geq 0, \\
C_{2} & :=\mathrm{SNR}^{\beta_{2}}, \beta_{2} \geq 0, \\
C_{1} & :=\mathrm{SNR}^{\beta_{1}}, \beta_{1} \geq 0,
\end{aligned}
$$

where $\beta_{3}$ and $\beta_{4}$ measure the strength of the interference/cross links compared to the direct links (which are assumed to be equal), while $\beta_{1}$ and $\beta_{2}$ the strength of the cooperation links compared to the direct links. The $\mathrm{gDoF}$ is defined as [8]

$$
\mathrm{d}:=\lim _{\mathrm{SNR} \rightarrow+\infty} \frac{\max \left\{R_{1}+R_{2}\right\}}{2 \log (1+\mathrm{SNR})}
$$

where the maximization is intended over all possible achievable rate pairs $\left(R_{1}, R_{2}\right)$.

The gDoF of the causal cognitive IC is lower bounded by the $\mathrm{gDoF}$ of the noncooperative IC and upper bounded by the $\mathrm{gDoF}$ of the non-causal cognitive IC. The $\mathrm{gDoF}$ of the noncooperative IC [8] (i.e., $\beta_{1}=\beta_{2}=0$ ) with $\beta_{3}=\beta_{4} \geq 0$ is the so-called W-curve [8], and that of the noncooperative Z-IC (which coincides with the S-IC) with $\beta_{3}=0$ and $\beta_{4} \geq 0$ is the minimum between the so-called V-curve [19] and $1 / 2$. The $\mathrm{gDoF}$ of the non-causal cognitive IC [9] (i.e., $\beta_{1}=0$ and $\left.\beta_{2}=+\infty\right)$ with $\beta_{3}=\beta_{4} \geq 0$ coincides with the V-curve, that of the Z-channel with $\beta_{3} \geq 0$ and $\beta_{4}=0$ is also the V-curve, and that of the S-channel with $\beta_{3}=0$ and $\beta_{4} \geq 0$ equals 1 .

The $\mathrm{gDoF}$ region is an asymptotically exact characterization of capacity at infinite SNR. At finite SNR the capacity is said to be known to within $\mathrm{b}$ bits if we can show an inner bound region $\mathcal{I}$ and an outer bound region $\mathcal{O}$ such that $\left(R_{1}, R_{2}\right) \in$ ConvexClosure $[\mathcal{I}] \Longrightarrow\left(R_{1}+\mathrm{b}, R_{2}+\mathrm{b}\right) \notin \mathcal{O}$.

The capacity of the IC with unilateral cooperation is sandwiched between that of the noncooperative IC [8] (i.e., $Y_{1}=Y_{2}=\emptyset$ ) and that of the non-causal cognitive IC [9] (i.e., $Y_{1}=\emptyset$ and $Y_{2}$ is a noiseless bit-pipe of infinite capacity), which are both known to within 1 bit.

In this work we focus on the interference-asymmetric IC with unilateral source cooperation obtained as

$$
\begin{aligned}
& \text { Z-channel: } \beta_{1}=0, \beta_{2}=\beta_{f}, \beta_{3}=\beta_{i}, \beta_{4}=0, \\
& \text { S-channel: } \beta_{1}=0, \beta_{2}=\beta_{f}, \beta_{3}=0, \beta_{4}=\beta_{i}
\end{aligned}
$$

for some non-negative pair $\left(\beta_{i}, \beta_{f}\right)$, which we will compare with the interference-symmetric IC, i.e., $\beta_{3}=\beta_{4}=\beta_{i}$, and the IC with symmetric bilateral source cooperation, i.e., $\beta_{1}=$ $\beta_{2}=\beta_{f}$.

\section{GDOF AND CONSTANT GAP FOR INTERFERENCE-SYMMETRIC CHANNELS: SUMMARY OF KNOWN RESULTS}

\section{A. FD Bilateral Cooperation}

Thm. 1 reported next is from [13]. In [13] a smaller gap compared to [4] was obtained. Note that [4] considered symmetric cooperation links $C_{1}=C_{2}$ but general $\left(S_{3}, I_{3}, S_{4}, I_{4}\right)$, while [13] only considered the case $C_{1}=C_{2}, S_{3}=S_{4}, I_{3}=$ $I_{4}$, which justifies the smaller gap.

Theorem 1 ([13]) The sum-capacity of the interferencesymmetric G-IC with bilateral FD source cooperation in known to within 4 bits.

Fig. 2(a) shows the gDoF and the gap for the interferencesymmetric G-IC with bilateral FD source cooperation. The whole set of parameters $\left(\beta_{i}, \beta_{f}\right)$ has been partitioned into multiple sub-regions depending upon different levels of cooperation $\left(\beta_{f}\right)$ and interference $\left(\beta_{i}\right)$. For each sub-region, the gDoF and constant gap results of [13] are reported.

By observing Fig. 2(a), we notice that cooperation might be worth implementing only in regions 5, 9 and 10, i.e., in the regimes where the $\mathrm{gDoF}$ is a function of $\beta_{f}$. In the strong interference regime, i.e., $1 \leq \beta_{i} \leq 2$, source 2 may use the information learned through the noisy link to help source 1 to convey the message to receiver 1 . In the weak interference regime, i.e., $\beta_{i}<1$, the acquired information may be exploited to pre-code the message intended to receiver 2 by against the interference created by the learned message.

\section{B. HD Bilateral Cooperation}

Thm. 2 reported next is an extension to the case of random switch of the result first obtained in [5, Thm.3.1] for the case of deterministic switch. In addition, the $\mathrm{gDoF}$ in [5] were given implicitly as the solution of a linear program, while here we give the $\mathrm{gDoF}$ in closed-form.

\section{Theorem 2 (random switch extension of [5, Thm.3.1])}

The sum-capacity of the interference-symmetric G-IC with bilateral $H D$ source cooperation in known to within 26.011 bits.

The proof parallels that of [20] (for the case of interferencesymmetric unilateral HD cooperation and random switch) and is not reported here for sake of space. In [5, Thm.3.1], the difference between upper and lower bounds gives a gap of $17+3=20$ bits on the symmetric sum-capacity. With random switch at each of the two sources, the sum-rate upper bound is expected to be increased by $2 \times \log (2)=2$ bits (since each source can convey at most one bit of information through a binary switch), thereby suggesting that the gap should be of 22 bits. However, we used the bound from [4, page 179, bottom of 1st column]; this bound contains six entropy terms with positive sign, each of them contributes to one extra bit to the gap; for this reason our gap is about 6 bits larger than the one in [5, Thm.3.1].

Fig. 2(b) shows the gDoF and the gap for the interferencesymmetric G-IC with bilateral HD source cooperation. The whole set of parameters has been partitioned into multiple subregions depending upon different levels of cooperation $\left(\beta_{f}\right)$ and interference $\left(\beta_{i}\right)$ strengths. For each sub-region, the gDoF and constant gap results are reported.

By comparing Fig. 2(b) with Fig. 2(a), we see that there exist parameter regimes where the gDoF of the HD channel equals that of the FD channel. This happens when the gDoF of 


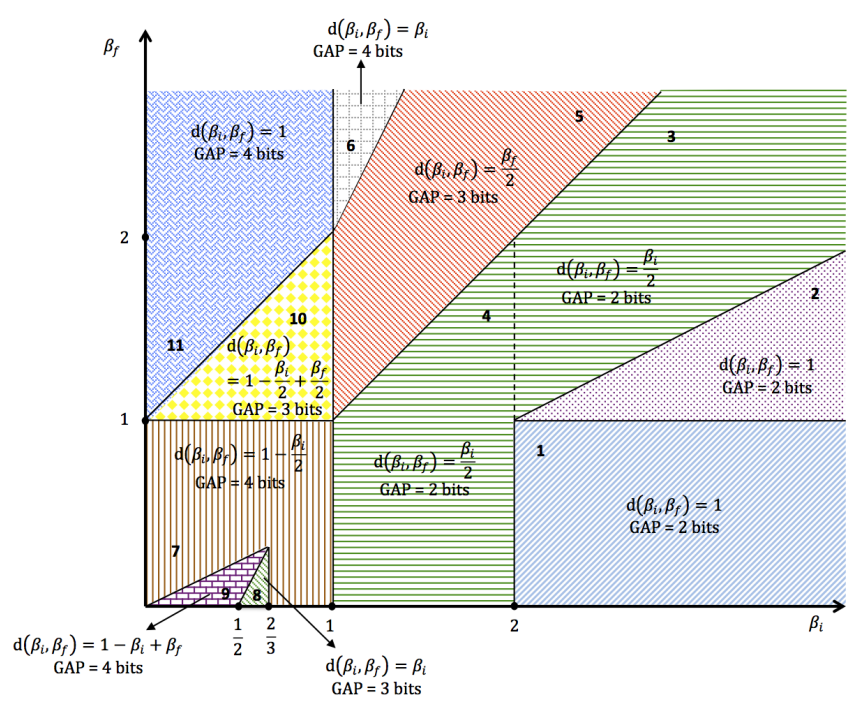

(a) Bilateral FD source cooperation.

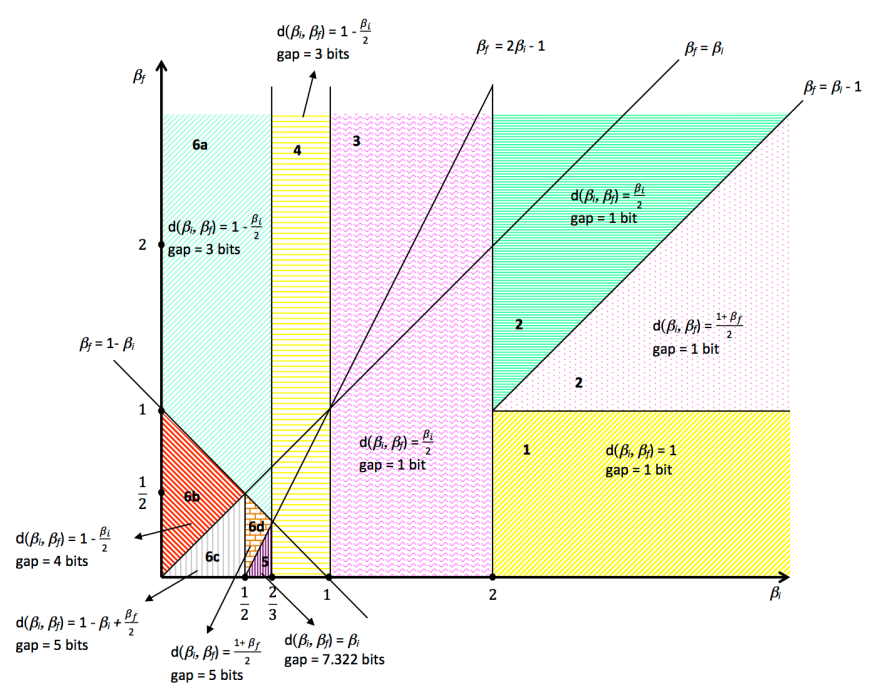

(c) Unilateral FD source cooperation.

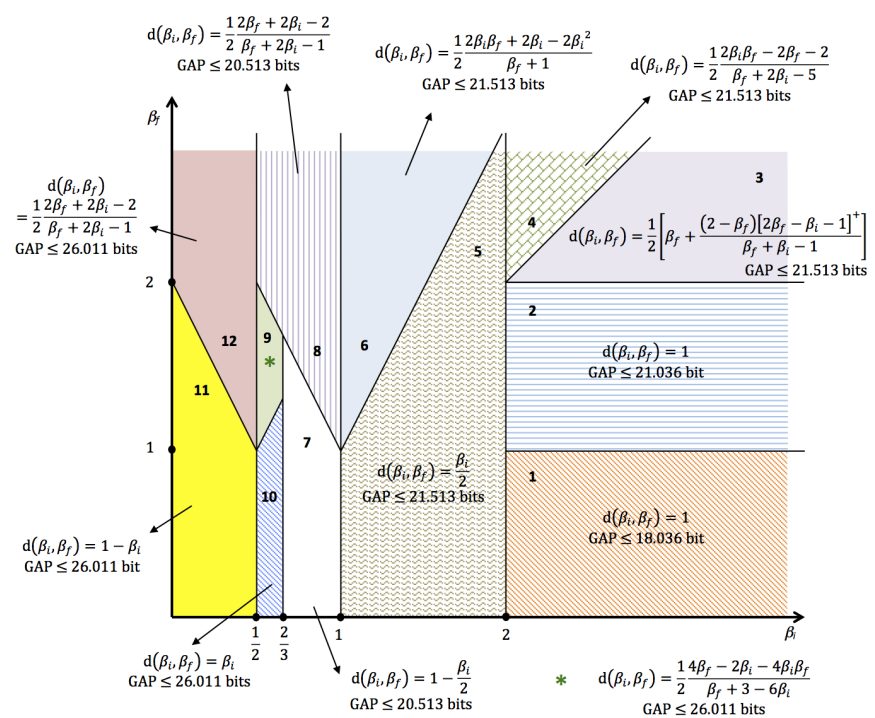

(b) Bilateral HD source cooperation.

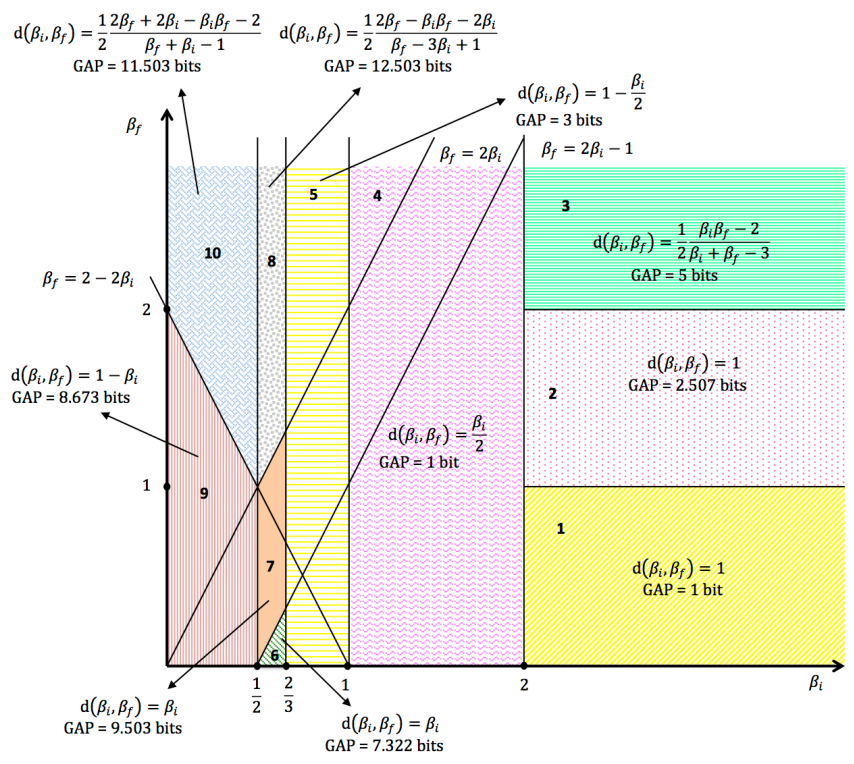

(d) Unilateral HD source cooperation.

Fig. 2: gDoF and constant gap results for interference-symmetric G-IC with source cooperation, either bilateral (cases (a) and (b)) or unilateral (cases (c) and (d)).

the system is the same as that of the noncooperative IC. Thus the same additive gap result found for the FD case holds in the case of HD cooperation. As an example consider the region 1 where $\beta_{i} \geq 2$ and $\beta_{f} \leq 1$, i.e., very strong interference and weak cooperation; in this regime we have $d=1$, for both FD and HD, thus the gap computed for the HD case (18.036 bits) can be reduced to the one obtained for the FD case (2 bits).

\section{FD Unilateral Cooperation}

Thm. 3 reported next is from [21]. In [21], we specialized the outer bounds of [2], [4], [11] to the case of unilateral cooperation (i.e., by setting $C_{1}=0$ ) and developed achievable schemes based on 'Block Markov superposition coding with joint decoding' and found that
Theorem 3 ([21]) The sum-capacity of the interferencesymmetric G-IC with unilateral FD source cooperation in known to within 7.322 bits.

A detailed proof can be found in [6].

Fig. 2(c) shows the gDoF and the gap for the symmetric G-IC with unilateral FD source cooperation. The whole set of parameters has been partitioned into multiple sub-regions depending upon different levels of cooperation $\left(\beta_{f}\right)$ and interference $\left(\beta_{i}\right)$ strengths. We notice that:

1) Unilateral source cooperation has the same $g D o F$ of the classical noncooperative IC (given by $\min \{\max \{1-$ $\left.\left.\left.\beta_{i}, \beta_{i}\right\}, \max \left\{1-\beta_{i} / 2, \beta_{i} / 2\right\}, 1\right\}\right)$ in regions $1,3,4$ and 5 in Fig. 2(c). For this set of parameters unilateral 
cooperation/causal cognitive radio might not be worth implementing in practical systems since the same gDoF is achieved without cooperation.

2) Unilateral source cooperation has the same $\mathrm{gDoF}$ of the classical relay channel (given by $\left.1 / 2 \max \left\{1, \min \left\{\beta_{i}, \beta_{f}\right\}\right\}\right)$ for $\beta_{i} \geq 1$ and $\beta_{f} \geq \beta_{i}$, i.e., part of regions 2 and 3 in Fig. 2(c). For this set of parameters unilateral cooperation/causal cognitive radio might not be worth implementing in practical systems since zero rate is optimal for the cognitive user.

3) Unilateral cooperation behaves differently than other classical channel models in terms of $\mathrm{gDoF}$ only in part of region $2\left(\beta_{f}<\beta_{i}\right)$ and region 6 in Fig. 2(c). Region 6 requires more involved achievable schemes , i.e., Dirty-Paper-Coding (DPC), and points to a tradeoff between constant gap and complexity. In [6] we showed that a DPC-based scheme achieves the sum-capacity to within 2 bits, rather than the 3 bits obtained by using superposition coding only.

4) Unilateral source cooperation has the same gDoF of the non-causal cognitive channel (given by $\min \{1-$ $\left.\beta_{i} / 2, \beta_{i} / 2\right\}$ ) in regions 2 (only for $\beta_{f} \geq \beta_{i}-1$ ), 3, 4, 6a, and $6 \mathrm{~b}$ in Fig. 2(c). Therefore, in these regimes, unilateral source cooperation attains the ultimate performance limits of the non-causal cognitive radio and is strictly better (in terms of $\mathrm{gDoF}$ ) than the noncooperative case.

5) Unilateral source cooperation has the same $\mathrm{gDoF}$ of bilateral source cooperation (see Fig. 2(a)) when $\beta_{f} \leq$ 1 or $\beta_{f} \in\left[\left[\beta_{i}-1\right]^{+}, \beta_{i}\right]$ except in the regimes $6 \mathrm{c}$ and $6 \mathrm{~d}$ in Fig. 2(c). For this set of parameters unilateral cooperation attains the same $\mathrm{gDoF}$ of bilateral cooperation but with less resources and therefore represents a better trade-off in practical systems.

\section{HD Unilateral Cooperation}

Thm. 4 reported next is from [20]. In [20], we specialized the FD outer bounds of [2], [4], [11] to the case of unilateral HD cooperation by following the approach of [15]. We also developed achievable schemes inspired by linear deterministic approximation of the Gaussian noise channel at high SNR [22] and found that

Theorem 4 ([20]) The sum-capacity of the interferencesymmetric G-IC with unilateral HD source cooperation in known to within 12.503 bits.

Fig. 2(d) shows the gDoF and the gap for the interferencesymmetric G-IC with unilateral HD source cooperation. The whole set of parameters has been partitioned into multiple subregions depending upon different levels of cooperation $\left(\beta_{f}\right)$ and interference $\left(\beta_{i}\right)$ strengths. We notice that:

1) Everywhere, except in regions 3,8 and 10 in Fig. 2(d), unilateral cooperation might not be worth implementing since the same $\mathrm{gDoF}$ is achieved without cooperation (classical IC).

2) Differently from the FD case, we notice that the gDoF of the IC with unilateral cooperation never equals the
gDoF of the HD relay channel [23]. In other words, the $\mathrm{gDoF}$ of the HD relay channel is always a strict lower bound for the IC with unilateral cooperation.

3) The IC with unilateral HD source cooperation has the same $\mathrm{gDoF}$ of the non-causal cognitive channel in regions 4 and 5 in Fig. 2(d). Thus, in these two regions, the performance of the system in terms of $\mathrm{gDoF}$ is not worsened by allowing causal learning at one source.

4) In regions 1, 4, 5 and 6 of Fig. 2(d) the gDoF equals that of the equivalent FD channel. Thus, in these regimes, the same gap results found for the FD case hold in the case of unilateral HD source cooperation.

5) In regions $1,2,6,7,9$, part of 5 (with $\beta_{f} \leq 3-2 \beta_{i}$ ) and part of 4 (with $\beta_{f} \leq 2 \beta_{i}-1$ ) of Fig. 2(d), the $\mathrm{gDoF}$ is the same as that computed in the bilateral case. Thus, for this set of parameters, unilateral cooperation attains the same gDoF of bilateral cooperation but with less resources and therefore represents a better trade-off in practical systems.

\section{GDOF AND CONSTANT GAP FOR INTERFERENCE-ASYMMETRIC CHANNELS: NEW RESULTS}

\section{A. Z-Channel: FD Unilateral Cooperation}

For the Z-channel with unilateral cooperation, obtained from the general G-IC model by setting $C_{1}=I_{4}=0$, we have:

Theorem 5 The sum-capacity in known to within 1 bit for the Z-G-IC with unilateral FD source cooperation.

The proof can be found in [6].

Fig. 3(a) shows the gDoF and the gap for the Z-G-IC with unilateral FD source cooperation. The whole set of parameters has been partitioned into multiple sub-regions depending upon different levels of cooperation $\left(\beta_{f}\right)$ and interference $\left(\beta_{i}\right)$ strengths. We notice that:

1) With no-cooperation we immediately have $\mathrm{d} \geq$ $\min \left\{1, \max \left\{\frac{\beta_{i}}{2}, 1-\frac{\beta_{i}}{2}\right\}\right\}$ from [7]. Thus, for the Zchannel, cooperation improves the $\mathrm{gDoF}$ with respect to the noncooperative case in the regime $\beta_{i} \geq 2$ and $\beta_{f} \geq 1$ (regions 2 and 3 in Fig. 3(a)).

2) Unilateral cooperation, for the Z-channel, attains the same gDoF of the relay channel when $1 \leq \beta_{i} \leq \beta_{f}$ (part of regions 3 and 4 in Fig. 3(a)).

3) The Z-channel achieves the same gDoF of the noncausal cognitive channel, given by $\mathrm{d}=\max \{1-$ $\left.\beta_{i} / 2, \beta_{i} / 2\right\}$, everywhere except in $\beta_{i}>\max \left\{2, \beta_{f}+1\right\}$ (regions 1 and 2 in Fig. 3(a)).

4) The gDoF of unilateral cooperation equals that of bilateral cooperation when $\beta_{f} \leq \max \left\{1, \beta_{i}\right\}$ (regions 1 and 2, parts of regions 3, 4 and 5 in Fig. 3(a)).

5) By comparing Fig. 2(c) and Fig. 3(a), we observe that the $\mathrm{gDoF}$ of the $\mathrm{Z}$-channel is always greater or equal than that of the symmetric G-IC with unilateral source cooperation. This is due to the fact that the source 1 does not cooperate in sending the signal of source 2 . Therefore by removing the link between source 1 and destination 2 we rid destination 2 of only interfering 


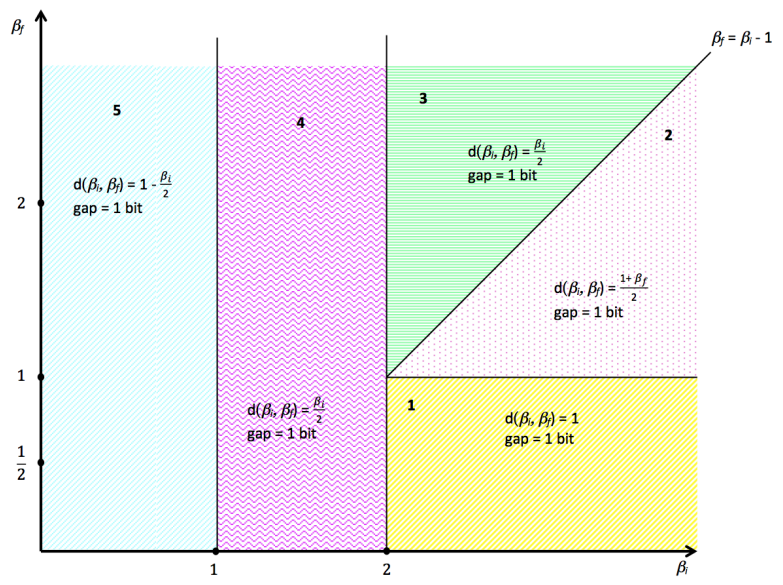

(a) Z-IC with unilateral FD cooperation.

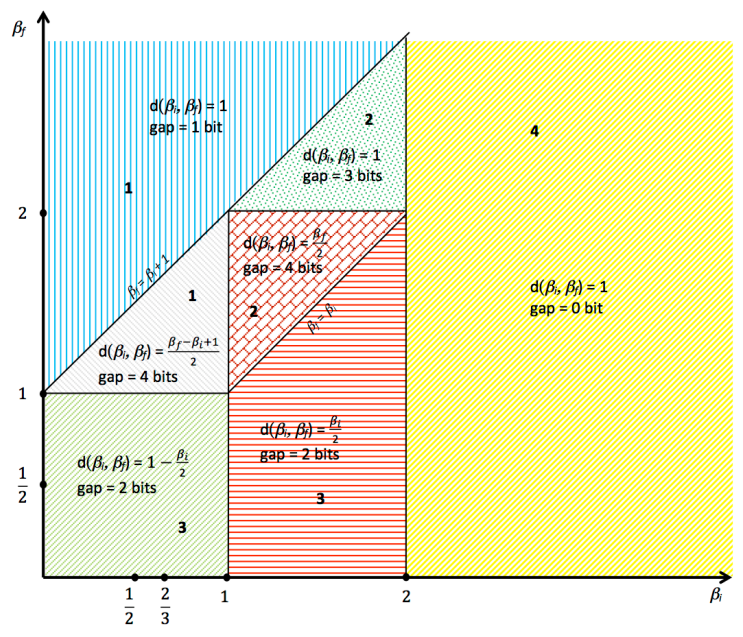

(c) S-IC with unilateral FD cooperation.

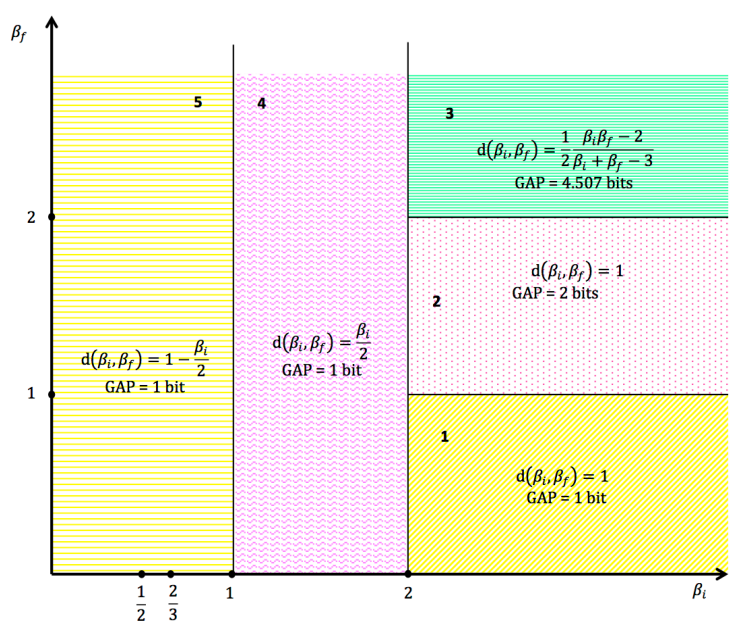

(b) Z-IC with unilateral HD cooperation.

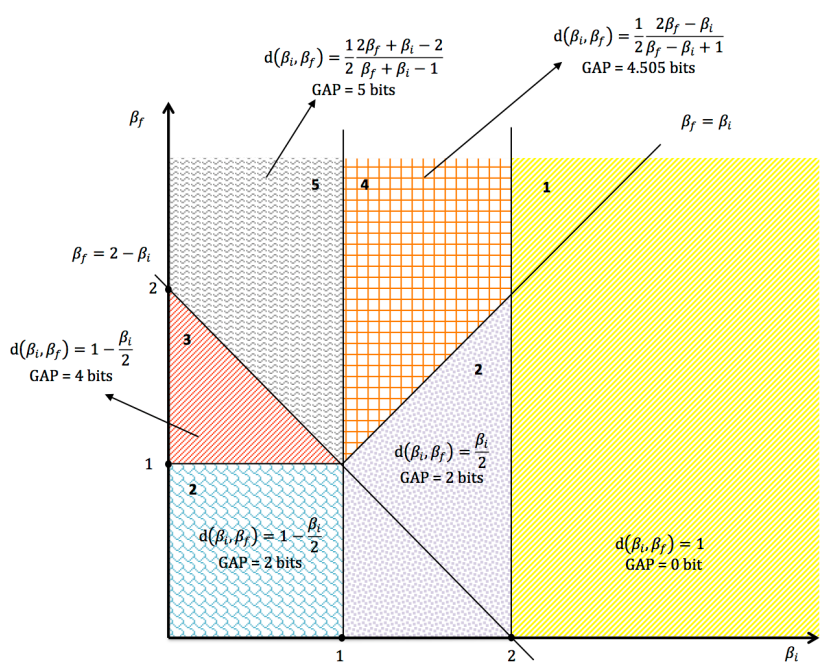

(d) S-IC with unilateral HD cooperation.

Fig. 3: gDoF and constant gap results for interference-asymmetric G-IC with unilateral source cooperation.

signal. The regimes where the Z-channel outperforms the symmetric channel are when $0 \leq \beta_{i} \leq \frac{2}{3}$ and $\beta_{f} \leq$ $\min \left\{\beta_{i}, 1-\beta_{i}\right\}$ (part of region 5 in Fig. 3(a)).

\section{B. Z-Channel: HD Unilateral Cooperation}

Thm. 6 reported next is an extension to the case of random switch of [5, Thm.3.2] for the case of deterministic switch. In addition, the $\mathrm{gDoF}$ in [5] were given implicitly as the solution of a linear program, while here we give the gDoF in closedform. Moreover, here we substantially reduce the gap from 20 bits to 4.507 bits:

Theorem 6 The sum-capacity in known to within 4.507 bits for the Z-G-IC with unilateral HD source cooperation.

The proof parallels that of [20] (for the case of interferencesymmetric unilateral HD cooperation and random switch).

Fig. 3(b) shows the gDoF and the gap for the Z-G-IC with unilateral HD source cooperation. The whole set of parameters has been partitioned into multiple sub-regions depending upon different levels of cooperation $\left(\beta_{f}\right)$ and interference $\left(\beta_{i}\right)$ strengths. The analysis is similar to that of the channel with symmetric interfering links in the same regimes. We notice:

1) By comparing Fig. 3(b) with Fig. 3(a), we notice that in regions 1, 4 and 5 of Fig. 3(b) the $\mathrm{gDoF}$ of the HD channel is as that of the FD. Moreover this happens when the performance of the system in terms of $\mathrm{gDoF}$ is not enhanced by allowing source cooperation. Thus the same gap result found for the FD case holds in the case of HD cooperation. Moreover, in region 2 in Fig. 3(b) the gDoF equals to that of the noncooperative IC.

2) Differently from the FD case, we notice that the $\mathrm{gDoF}$ of the Z-G-IC with unilateral source cooperation never equals the $\mathrm{gDoF}$ of the classical HD relay channel [23].

3) The Z-channel achieves the same gDoF of the noncausal cognitive channel everywhere except in $\beta_{i}>2$ (regions 1, 2 and 3 in Fig. 3(b)).

4) By comparing Fig. 3(b) and Fig. 2(d) we observe that 
the Z-channel outperforms the symmetric channel when $0 \leq \beta_{i} \leq \frac{2}{3}$ (part of region 5 in Fig. 3(b)). The reason of this behavior is the same as the one stated for the FD case.

\section{S-Channel: FD Unilateral Cooperation}

For the S-channel with unilateral cooperation, obtained from the general G-IC model by setting $C_{1}=I_{3}=0$, we have:

Theorem 7 The sum-capacity in known to within 4 bits for the S-G-IC with unilateral FD source cooperation.

The proof can can be found in [6].

Fig. 3(c) shows the gDoF and the gap for the G-S channel with unilateral FD source cooperation. The whole set of parameters has been partitioned into multiple sub-regions depending upon different levels of cooperation $\left(\beta_{f}\right)$ and interference $\left(\beta_{i}\right)$ strengths. In [6], we developed achievable schemes. In contrast to the symmetric and $\mathrm{Z}$ channels, here we made use of DPC-based schemes in regions 1 and 2 of Fig. 3(c). We notice that:

1) Unilateral cooperation achieves the same $\mathrm{gDoF}$ of the noncooperative IC when $\beta_{i} \geq 2$ or $\beta_{f} \leq \max \left\{1, \beta_{i}\right\}$ (regions 3 and 4 in Fig. 3(c)).

2) The gDoF of unilateral cooperation never equals the $\mathrm{gDoF}$ of the classical relay channel, which is equal to $\mathrm{d}=\frac{1}{2}$.

3) The S-channel achieves the $\mathrm{gDoF}$ of the non-causal cognitive IC everywhere except in $\beta_{i} \leq 2$ and $\beta_{f} \leq \min \left\{2, \beta_{i}+1\right\}$ (regions 1, 3 and part of region 2 in Fig. 3(c)). Actually, when the link source $2 \rightarrow$ destination 1 is not present, the non-causal cognitive IC achieves $d=1$, i.e., the performance in terms of $\mathrm{gDoF}$ is the same as that of a two point-to-point communication channel. This is so because the cognitive source, by a-priori knowing the primary's message, can completely pre-cancel the interference experienced at its receiver.

4) The gDoF of unilateral and bilateral cooperation are equal when $\beta_{i} \geq 2$ and $\beta_{f} \leq 1$ or when $\beta_{i} \leq 2$ and $\beta_{f} \leq \min \left\{2, \beta_{i}+1\right\}$ (regions 1 and 3 , and parts of regions 2 and 4 in Fig. 3(c)).

5) The S-channel outperforms the interference-symmetric IC with unilateral source cooperation when either $0 \leq$ $\beta_{i} \leq \frac{2}{3}$ and $\beta_{f} \leq \min \left\{\beta_{i}, 1-\beta_{i}\right\}$ or when $\beta_{i} \leq 2$ and $\beta_{f} \geq \max \left\{1, \beta_{i}\right\}$ (regions 1,2 and part of region 3 in Fig. 3(c)). On the other hand, the interference-symmetric IC with unilateral source cooperation outperforms the $\mathrm{S}$ channel when $\beta_{i} \geq 2$ and $\beta_{f} \geq 1$ (part of region 4 in Fig. 3(c)). This is so because, in the very strong interference and strong cooperation regime, the performance of the system is enhanced by allowing the cognitive source to help the primary user to convey the information, but this is not possible since $I_{3}=0$.

6) When $\beta_{i} \geq 2$ (region 4 in Fig. 3(c)) we have an exact sum-capacity result, i.e., the gap between the sum-rate outer bound and inner bound is equal to zero.

\section{S-Channel: HD Unilateral Cooperation}

Thm. 8 reported next is an extension to the case of random switch of [5, Thm.3.2] for the case of deterministic switch. In addition, the $\mathrm{gDoF}$ in [5] were given implicitly as the solution of a linear program, while here we give the $\mathrm{gDoF}$ in closedform. Moreover, here we substantially reduce the gap from 20 bits to 5 bits:

Theorem 8 The sum-capacity in known to within 5 bits for the $S$-G-IC with unilateral HD source cooperation.

The proof parallels that of [20] (for the case of interferencesymmetric unilateral HD cooperation and random switch).

Fig. 3(d) shows the gDoF and the gap for the G-S channel with unilateral HD source cooperation. The whole set of parameters has been partitioned into multiple sub-regions depending upon different levels of cooperation $\left(\beta_{f}\right)$ and interference $\left(\beta_{i}\right)$ strengths. We notice that:

1) By comparing Fig. 3(d) with Fig. 3(c), we notice that there are some regions (3 and 4 in Fig. 3(c)) in which the $\mathrm{gDoF}$ of the HD channel is as that of the FD channel. This happens when the gDoF of the system is the same as that of the pure IC. Thus, in these regions, the same additive gap results found for the FD case hold in the case of HD cooperation. Moreover, in region 3 in Fig. $3(d)$ the gDoF equals to that of the pure IC.

2) As obtained for the FD case, also the gDoF of the S-GIC with unilateral HD source cooperation never equals that of the classical HD relay channel given by $d=\frac{1}{2}$.

3) The S-channel achieves the same $\mathrm{gDoF}$ of the non-causal cognitive channel, that is, $d=1$, for $\beta_{i} \geq 2$ (region 1 in Fig. 3(d)).

4) The S-channel outperforms the interference-symmetric IC with unilateral source cooperation when either $0 \leq$ $\beta_{i} \leq \frac{2}{3}$ or when $\beta_{i} \leq 2$ and $\beta_{f} \geq \max \left\{2-\beta_{i}, \beta_{i}\right\}$ (regions 4 and 5 , and parts of regions 2 and 3 in Fig. 3(d)). On the other hand, the interference-symmetric IC with unilateral source cooperation outperforms the S-channel in very strong interference and very strong cooperation, i.e., $\beta_{i} \geq 2$ and $\beta_{f} \geq 2$ (region 2 in Fig. 3(d)). The reason of this behavior is the same as the one explained for the FD case.

5) When $\beta_{i} \geq 2$ (region 1 in Fig. 3(d)), i.e. very strong interference regime, we have an exact sum-capacity result, as in the FD case.

\section{CONCLUSIONS}

In this work we considered the two-user Gaussian interference channel with unilateral source cooperation and with asymmetric interference. Our main contribution consisted in deriving closed-form expressions for the $\mathrm{gDoF}$ and characterizing the sum-capacity to within a constant gap for both cases where the cognitive source operates in full-duplex and half-duplex mode.

We also systematically compared the different channel models among themselves and with the case of: (i) bilateral symmetric cooperation and interference, (ii) unilateral cooperation 
and symmetric interference, (iii) non-causal cognitive interference channel, (iv) noncooperative interference channel and (v) relay channel. In particular we highlighted regimes where cooperation might not be worth implementing in practical systems (because approximately equal to that of the relay channel or of the noncooperative channel), as well as regimes where causal cognition approximately equals the sum-capacity of the non-causal cognitive channel. Moreover, by comparing the symmetric-interference case with the asymmetric-interference case we discussed the impact on the sum-capacity of the network topology.

\section{ACKNOWLEDGMENT}

The work of Dr. D. Tuninetti was partially funded by NSF under award number 0643954; the contents of this article are solely the responsibility of the author and do not necessarily represent the official views of the NSF. The work of Dr. D. Tuninetti was possible thanks to the generous support of Telecom-ParisTech, Paris France, while the author was on a sabbatical leave at the same institution.

Eurecom's research is partially supported by its industrial partners: BMW Group Research \& Technology, IABG, Monaco Telecom, Orange, SAP, SFR, ST Microelectronics, Swisscom and Symantec. The research carried out at Eurecom leading to these results has received funding from the EU Celtic+ Framework Program Project SPECTRA.

The research at IMC leading to these results has received funding from the European Union Seventh Framework Programme (FP7/2007-2013) under grant agreement iJOIN n 317941 .

\section{REFERENCES}

[1] N. Devroye, P. Mitran, and V. Tarokh, "Achievable rates in cognitive radio channels," IEEE Trans. on Info. Theory, vol. 52, no. 5, pp. $1813-$ 1827, May 2006.

[2] A. Host-Madsen, "Capacity bounds for cooperative diversity," IEEE Trans. on Info. Theory, vol. 52, no. 4, pp. 1522-1544, April 2006.

[3] S. Yang and D. Tuninetti, "Interference channel with generalized feedback (a.k.a. with source cooperation): Part i: Achievable region," IEEE Trans. on Info. Theory, vol. 57, no. 5, pp. 2686-2710, May 2011.

[4] V. Prabhakaran and P. Viswanath, "Interference channels with source cooperation," IEEE Trans. on Info. Theory, vol. 57, no. 1, pp. 156-186, Jan. 2011

[5] R. Wu, "Interference channels with half-duplex source cooperation," Master's thesis, Electrical and Computer Eng. Dept., University of Illinois at Urbana-Champaign, May 2011. [Online]. Available: http://hdl.handle.net/2142/24136

[6] M. Cardone, D. Tuninetti, R. Knopp, and U. Salim, "On the gaussian interference channel with causal cognition, or with unilateral source cooperation," submitted to IEEE Transactions on Information Theory in Nov 2012, available at arxiv:1207.5319, 2012.

[7] I. Sason, "On achievable rate regions for the gaussian interference channel," IEEE Trans. on Info. Theory, vol. 50, no. 6, pp. 1345-1356, June 2004.

[8] R. Etkin, D. Tse, and H. Wang, "Gaussian interference channel capacity to within one bit," IEEE Trans. on Info. Theory, vol. 54, no. 12, pp. 5534-5562, Dec. 2008.

[9] S. Rini, D. Tuninetti, and N. Devroye, "Inner and outer bounds for the gaussian cognitive interference channel and new capacity results," IEEE Trans. on Info. Theory, vol. 58, no. 2, pp. 820-848, Feb. 2012.

[10] E. Telatar and D. Tse, "Bounds on the capacity region of a class of interference channels," in IEEE Int. Symposium on Info. Theory (ISIT), june 2007, pp. 2871-2874.

[11] D. Tuninetti, "An outer bound region for interference channels with generalized feedback," in Information Theory and Applications Workshop (ITA), 2010, Feb. 2010, pp. 1-5.
[12] G. Kramer, "Outer bounds on the capacity of gaussian interference channels," IEEE Transactions on Information Theory, vol. 20, no. 3 , pp. 581-586, 2004.

[13] Y. Shuang (Echo), "On interference channels with generalized feedback," Ph.D. dissertation, ECE Department, University of Illinois at Chicago, Chicago, August 2010.

[14] M. Mirmohseni, B. Akhbari, and M. Aref, "On the capacity of interference channel with causal and noncausal generalized feedback at the cognitive transmitter," IEEE Trans. on Info. Theory, vol. 58, no. 5, pp. 2813-2837, May 2012.

[15] G. Kramer, "Models and theory for relay channels with receive constraints," in 42nd Annual Allerton Conf. on Commun., Control, and Computing, 2004, pp. 1312-1321.

[16] Z. Wu and M. Vu, "Partial decode-forward binning schemes for the causal cognitive relay channels," arxiv: 1111.3966, 2011.

[17] S. Seyedmehdi, J. Jiang, Y. Xin, and X. Wang, "An improved achievable rate region for causal cognitive radio," in IEEE Int. Symposium on Info. Theory (ISIT), July 2009, pp. 611-615.

[18] Y. Cao and B. Chen, "The cognitive z channel," in 43rd Annual Conference on Information Sciences and Systems (CISS), March 2009, pp. $528-532$.

[19] C. Suh and D. Tse, "Feedback Capacity of the Gaussian Interference Channel to within 2 Bits," IEEE Trans. on Info. Theory, vol. 57, no. 5 , pp. 2667-2685, May 2011.

[20] M. Cardone, D. Tuninetti, R. Knopp, and U. Salim, "The symmetric sum-capacity of the gaussian half-duplex causal cognitive interference channel to within a constant gap," submitted to ISIT 2013, July 2013.

[21] - -, "On the interference channel with causal cognition," to appear in IEEE International Conference on Communications (ICC) 2013, July 2013.

[22] A. Avestimehr, S. Diggavi, and D. Tse, "Wireless network information flow: A deterministic approach," IEEE Trans. on Info. Theory, vol. 57, no. 4, pp. 1872-1905, 2011.

[23] M. Cardone, D. Tuninetti, R. Knopp, and U. Salim, "Gaussian halfduplex relay channels: Generalized degrees of freedom and constant gap results," to appear in IEEE International Conference on Communications (ICC), see also arXiv:1301.5522, July 2013. 